\section{Autoantikörper gegen oxidiertes LDL}

W. Stöcker und C. Krüger

Euroimmun Medizinische Labordiagnostika AG, Lübeck, Deutschland

Synonym(e) oxLDL-Antikörper

Englischer Begriff antibodies to oxidized LDL

Funktion - Pathophysiologie Oxidiertes $>$ Low density lipoprotein (LDL) stellt den Hauptanteil der in atherosklerotischen Läsionen enthaltenen Lipoproteine. Bei verschiedenen autoimmunen und chronisch entzündlichen Erkrankungen (systemischer Lupus erythematodes, Antiphospholipid-Syndrom, progressive Systemsklerose, Diabetes mellitus, Myokardinfarkt) konnten Komplexe aus oxidiertem LDL (oxLDL) und dem Phospholipid-bindenden Plasmaprotein $\beta 2$-Glykoprotein I ( $\beta 2 \mathrm{GPI}$ ) nachgewiesen werden.

Untersuchungsmaterial Serum, Plasma.

Probenstabilität Autoantikörper sind bei $+4{ }^{\circ} \mathrm{C}$ bis $\mathrm{zu}$ 2 Wochen lang beständig, bei $-20^{\circ} \mathrm{C}$ über Monate und Jahre hinweg.
Analytik Bisher sind Autoantikörper gegen oxidiertes LDL sowie gegen den Komplex aus oxLDL/ß2GPI beschrieben. Sie werden mittels $>$ Enzyme-linked Immunosorbent Assay bestimmt.

Indikation Möglicherweise Risikoeinschätzung arterieller Thrombosen bei Patienten mit systemischem Lupus erythematodes und Antiphospholipid-Syndrom sowie generell als Marker der Progression einer Atherosklerose, vor allem in Zusammenhang mit verschiedenen autoimmunen und chronisch entzündlichen Erkrankungen.

Diagnostische Wertigkeit Autoantikörper gegen oxLDL/ 32GPI wurden bei Patienten mit systemischem Lupus erythematodes sowie mit Antiphospholipid-Syndrom gefunden. Sie weisen auf arterielle Thrombosen hin. Ob diese Autoantikörper das Potenzial eines prognostischen Markers besitzen, ist Gegenstand der Forschung.

\section{Literatur}

Salonen JT, Yla-Herttuala S, Yamamoto R et al (1992) Autoantibody against oxidised LDL and progression of carotid atherosclerosis. Lancet 339:883-887 